\title{
Effective-medium model for nearly constant loss in ionic conductors
}

\author{
J. Ross Macdonald ${ }^{\text {a) }}$ \\ Department of Physics and Astronomy, University of North Carolina, Chapel Hill, \\ North Carolina 27599-3255
}

(Received 28 October 2002; accepted 27 March 2003)

\begin{abstract}
A complex quantitative model for nearly constant loss (NCL) is proposed based on an effective-medium approach. Unlike previous NCL response models, it satisfies the Kronig-Kramers transform relations. Here the effective-medium dielectric-level model depends directly on the concentration of mobile charge present and its complex dielectric response is identified as arising from electrical interactions between vibrating and/or hopping ions and the bulk matrix material. The parallel combination of the effective-medium response with dispersive hopping described by the Kohlrausch K1 model, a version of the corrected-modulus-formalism approach, leads to behavior that can represent dominant NCL at low temperatures well and, at higher temperatures, dispersive response followed by NCL. Complex nonlinear-least-squares fitting of experimental data sets that exhibit both types of response leads to excellent fits. Further, the effective-medium NCL model, which involves physically realizable response, can represent a wide range of NCL behavior analytically. Such behavior ranges from either approximate or exact power-law frequency dependence for both parts of the complex dielectric constant or to such response for its real part and very close to constant loss over a wide range of frequency for the associated imaginary part, as sometimes observed. (C) 2003 American Institute of Physics. [DOI: 10.1063/1.1576289]
\end{abstract}

\section{INTRODUCTION}

Although it has been known for many years that the real part of the ac electrical conductivity of ionic conductors often shows nearly linear frequency dependence at sufficiently low temperatures and/or high frequencies, only recently has this important phenomenon attracted widespread interest and led to much discussion and analysis. ${ }^{1-16}$ Such frequency dependence leads to nearly constant loss (NCL) at the complex dielectric constant level. The complex conductivity and the complex dielectric constant (or relative dielectric permittivity) may be written as $\sigma(\omega)=\sigma^{\prime}(\omega)+i \sigma^{\prime \prime}(\omega)$ and $\varepsilon(\omega)$ $=\varepsilon^{\prime}(\omega)-i \varepsilon^{\prime \prime}(\omega)=\sigma(\omega) / i \omega \varepsilon_{V}$, respectively, where $\varepsilon_{V}$ is the permittivity of vacuum.

We next define $\sigma_{S}^{\prime}(\omega) \equiv \sigma^{\prime}(\omega)-\sigma_{0}$ and $\varepsilon_{S}^{\prime \prime}(\omega)$ $\equiv \varepsilon^{\prime \prime}(\omega)-\left(\sigma_{0} / \omega \varepsilon_{V}\right)$, where $\sigma_{0}$ is the dc value of $\sigma^{\prime}(\omega)$. In the frequency region where $\sigma_{S}^{\prime}(\omega)$ is approximated well by $A \omega^{n}$, with $A$ frequency independent and $n$ slightly smaller than $1, \varepsilon_{S}^{\prime \prime}(\omega)$ will then be proportional to $\omega^{n-1}$, NCL behavior. For $n=1$, one obtains constant loss not physically plausible over an appreciable frequency range, ${ }^{5}$ although this has been a common choice (e.g., in Refs. 2-4, 8, and 11). One reason for this choice is that most of the prior work not by the present author has primarily dealt only with $\sigma^{\prime}(\omega)$ data, often does not employ even nonlinear-least-squares fitting, and never uses complex-nonlinear-least-squares (CNLS) fitting.

In such situations, the parameter $n$ is not accurately estimated and its value is not usually distinguished from unity. It can be well estimated, however, by using the free LEVM CNLS program. ${ }^{17}$ This program allows one to fit both the

\footnotetext{
${ }^{\text {a)} E l e c t r o n i c ~ m a i l: ~ m a c d @ e m a i l . u n c . e d u ~}$
}

real and imaginary parts of the data simultaneously to a complex response model, and it invariably yields estimates of $n$ slightly smaller than unity (see e.g., Refs. 5, 7, 12, and 16). For many thermally activated situations where NCL dominates, the terms involving $\sigma_{0}$ in the above equations are negligible compared to $A \omega^{n}$ response, both because the temperature dependence of $A$ is found to be far less than that of $\sigma_{0}$ and because in the high-frequency region $A \omega^{n}$ increases faster with increasing frequency than do the ordinary ionichopping dispersion effects present in the total response.

Most of the models used in discussing conductivesystem data that exhibit some NCL behavior have included in expressions for $\sigma^{\prime}(\omega)$ not only a part that represents the dispersive behavior associated with mobile charges but also a separate part, such as $A \omega^{n}$, to represent NCL. The $\log -\log$ slope (called slope hereafter) of $\sigma^{\prime}(\omega)$ will then increase towards $n$ as the frequency increases and the $A \omega^{n}$ term begins to dominate. We shall be concerned herein with more sophisticated versions of such composite-model approaches, ones with the NCL part associated with bulk dielectric effects as well as with ionic vibration and hopping. The generality of such composite models seems necessary to adequately represent both direct ionic-motion effects and indirect ones.

It is worth mentioning, however, that some models, such as the random barrier models of Dyre, ${ }^{6,18}$ although involving hopping behavior alone, lead to a $\sigma^{\prime}(\omega)$ slope that continuously increases toward unity as the frequency increases. Such unitary models do not generally lead to physically realistic low-frequency-limiting slopes of $\varepsilon_{S}^{\prime}(\omega)$ and $\varepsilon_{S}^{\prime \prime}(\omega)$, and they do not include the sort of dipolar-ion vibration interactions considered herein. Further, it does not seem that they can yield the NCL behavior often observed at suffi- 
ciently low temperatures where the temperature dependence of $\sigma^{\prime}(\omega)$ is much smaller than that of the ion-hopping thermally activated behavior observed at appreciably higher temperatures. ${ }^{1-3,7}$

In Sec. II, important composite models are defined and discussed, especially an effective-medium (EM) one for NCL. Then in Sec. III, accurate fits of experimental data are used to illustrate the extrapolated behavior of the EM part of the response model both for limited-range data that show only a small amount of NCL at high frequencies and for low-temperature data entirely dominated by NCL. Other NCL response models are discussed in Sec. IV, and Sec. V is a brief summary of conclusions.

\section{MODELING OF DISPERSIVE DATA}

\section{A. Background and earlier fitting elements and models}

The present story begins with the development in 1973 of the important original modulus formalism (OMF) for describing thermally activated dispersive frequency response of conductive systems. ${ }^{19}$ Although this model has been and continues to be widely used, it does not treat the highfrequency-limiting dielectric constant of the material, $\varepsilon_{\infty}$, properly, and thus it turns out to be both theoretically and experimentally inconsistent and inappropriate. ${ }^{20-26} \mathrm{We}$ denote the bulk dielectric constant as $\varepsilon_{D_{\infty}}$; it is found to be frequency independent over the range of usual experimental data when ionic conduction is negligible. As shown in Ref. 7, however, the total bulk dielectric constant seems to involve an additional small frequency-dependent term that increases with the concentration of mobile charge. The present work is primarily concerned with analysis of such behavior since it appears to offer a physically plausible explanation of NCL even though it seems to be a second-order effect.

The corrected modulus formalism model (CMF), like the OMF, involves Kohlrausch frequency response indirectly derived from the assumption of stretched-exponential temporal behavior. $^{7,19-26}$ We denote the model that involves such Kohlrausch response by $\mathrm{K} 1$, written as KWW1 in earlier work. It is used alone for the OMF, but early versions of the $\mathrm{CMF}$ approach involve both $\mathrm{K} 1$ and a dielectric constant $\varepsilon_{x}$ or capacitance in parallel; it has thus been denoted by CK1, ${ }^{5,7,12,24}$ a composite model, and leads to far better data fitting than does the OMF because it takes explicit account of $\varepsilon_{D \infty}$ by means of the separate free parameter $\varepsilon_{x}$. Then the high-frequency limiting dielectric constant is given by $\varepsilon_{\infty}$ $=\varepsilon_{D \infty}+\varepsilon_{C 1 \infty}$, where $\varepsilon_{C 1 \infty} \equiv \varepsilon_{C 1}(\infty)$ is the K1 nonzero high-frequency-limiting response that arises entirely from conductive-system mobile charge effects. ${ }^{7,24,26}$ Incidentally, the so-called Jonscher universal dynamic response expression widely used in $\sigma^{\prime}(\omega)$ for modeling ionic dispersion effects (see, e.g., Refs. 2 and 25) need not be considered here since its complex version has been shown to be much less appropriate for data fitting than the CK1 model..$^{25,26}$

Because we will be dealing herein with both individual response elements as well as with numerous composite models that involve such elements, Table I has been included to define, codify, and characterize them. In Table I, the
TABLE I. Summary of the model building blocks and composite models. Composite models are generally designated by names like PEMSE, where $\mathrm{M}$ is the model name, PE identifies a response element in parallel electrically with $\mathrm{M}$, and SE indicates an element in series with $\mathrm{M}$.

\begin{tabular}{llcc}
\hline $\begin{array}{l}\text { Block } \\
\text { No. }\end{array}$ & \multicolumn{1}{c}{$\begin{array}{c}\text { Response element or } \\
\text { model }\end{array}$} & $\begin{array}{c}\text { Number of } \\
\text { parameters }\end{array}$ & $\begin{array}{c}\text { Response } \\
\text { type }\end{array}$ \\
\hline $\begin{array}{c}\text { Model building } \\
1\end{array}$ & blocks & & \\
2 & C Capacitor & 1 & $\varepsilon_{D \infty}$ or blocking \\
3 & EM Effective medium & 3 or 4 & NCL \\
4 & K1 KWW1 & 3 & OMF \\
5 & P PCPE & 2 & NCL \\
Composite & S SCPE & 2 & Electrode response \\
models & & & \\
6 & CK1 & & CMF \\
7 & CK1S & 4 & CMF \\
8 & CP parallel & 6 & CMF \\
9 & CPK1 & 3 & CMCL \\
10 & EMC & 6 & CMF \\
11 & EMK1 & 4 or 5 & NCLing \\
12 & PC series & 6 or 7 & NCL, blocking \\
13 & PK1 & 3 & CMF \\
14 & PK1S & 7 & \\
\hline \hline
\end{tabular}

complex-response complex phase element $(\mathrm{CPE}),{ }^{27}$ has been denoted just by $\mathrm{P}$, which represents the PCPE when the CPE is in parallel with other elements and usually by S, the SCPE when the CPE response is in series with other response elements. An exception is the PC, a CPE in series with an ideal capacitor, which could be alternatively designated as SC. ${ }^{5}$ As discussed in Sec. II B, the present EM response involves a parameter $\eta$ that represents the relative concentration of mobile charges. The value of this parameter is usually known for a given material and it should then be held fixed, but since it can also be used as a free parameter the number of free parameters shown in Table I includes this possibility. CNLS fitting comparisons between the $\mathrm{K} 1$ and most of the composite models involving it listed in Table I appear in Ref. 26.

For situations where NCL appears to be present in the range of measurement, it has been found preferable to replace the parallel ideal dielectric constant of the CK1 by a lossy, complex-power-law expression, thus yielding the PK1 model. ${ }^{5,7,12,16,26}$ The PCPE part of this composite model may be expressed at the complex dielectric level by

$$
\varepsilon_{\mathrm{PC}} \equiv A_{\mathrm{PC}}(i \omega)^{-\gamma_{\mathrm{PC}}},
$$

where $0 \leqslant \gamma_{\mathrm{PC}} \leqslant 1$. Thus, when $\gamma_{\mathrm{PC}}=0, \varepsilon_{\mathrm{PC}}$ is an ordinary dielectric constant, and when $\gamma_{\mathrm{PC}}=1, \varepsilon_{\mathrm{PC}}$ leads to a pure conductance that would then contribute a term to $\sigma_{0}$. When $\varepsilon_{\mathrm{PC}}$ is employed to represent NCL behavior, $5,7,12,16 \gamma_{\mathrm{PC}} \ll 1$ and then $A_{\mathrm{PC}} \cong \varepsilon_{D_{\infty}}$. Finally, the CPK1 model has been used to fit data with the parallel $\mathrm{C}$ and $\mathrm{P}$ parts denoting $\varepsilon_{D \infty}$ and NCL effects, respectively, and, as usual, K1 represents the dispersive response associated only with ionic motion. ${ }^{7}$

\section{B. Effective-medium model for nearly constant loss}

Although the CP combination has been successfully employed to represent putative dielectric effects indirectly associated with ionic motion, ${ }^{5,7}$ it involves a simple sum of two 
dielectric effects and does not lead to a physically correct response at limiting low and high frequencies. These defects are banished or ameliorated if the two effects are combined using the physically plausible Maxwell-Garnett effectivemedium approximation. ${ }^{28-30}$ The EM approach “... is useful when one of the components can be considered as a host in which the inclusions of the other component are embedded." ${ }^{29}$ The host involves a homogeneous dielectric constant $\varepsilon_{0}$ and, in the simplest case, the inclusions are taken as spheres of dielectric constant $\varepsilon_{1}$ arranged on a cubic lattice. ${ }^{28}$ The resulting dielectric constant response, $\varepsilon_{\mathrm{EM}}$, may then be expressed as

$$
\varepsilon_{\mathrm{EM}} / \varepsilon_{0}=1-\frac{3 \eta\left(\varepsilon_{0}-\varepsilon_{1}\right)}{\left(2 \varepsilon_{0}+\varepsilon_{1}\right)+\eta\left(\varepsilon_{0}-\varepsilon_{1}\right)},
$$

where $\eta$ is the volume fraction of the inclusions. The composite model that involves EM and $\mathrm{K} 1$ responses in parallel, the EMK1, should be superior to the CPK1 model since it involves physically realizable limiting responses as well as additional generality. Both models may involve as many as six possibly free parameters when $\eta$ is taken as fixed. All the OMF and CMF models of Table I have been instantiated in LEVM and allow very accurate CNLS fitting.

The above expression involves dipolar but no higher multipole interactions among the inclusions, an excellent approximation when $\eta<0.4$, as shown in Ref. 28. Its limiting values are for $\eta=0, \varepsilon_{\mathrm{EM}}=\varepsilon_{\mathrm{EM}}^{\prime}=\varepsilon_{0}$, and for $\eta=1, \varepsilon_{\mathrm{EM}}^{\prime}$ $=\varepsilon_{1}$. For the present situation, we set $\varepsilon_{0}$ equal to the frequency-independent dielectric constant of the bulk in the absence of mobile ions, $\varepsilon_{D \infty}$. Note that it is inappropriate to define $\varepsilon_{0}$ as the full complex-dielectric response associated entirely with ionic motion, $\varepsilon_{C 1}(\omega)$, as in the K1 model, or as its limiting value $\varepsilon_{C 1 \infty}{ }^{7,30}$

Next, we follow the work in Ref. 7 by representing the dielectric effect of ions vibrating over a limited region, for example when localized in potential wells produced by surrounding bulk atoms and ions, as of lossy dipolar character. We then assume that the vibrating ions interact electrically with bulk dipoles and thus augment the bulk dielectric response. As a first approximation, it is plausible and consistent with previous work to approximate this dielectric effect of the vibrating ions by setting $\varepsilon_{1}=\varepsilon_{\mathrm{PC}}(\omega)$. We shall approximate $\eta$ here by $x_{c}$, the fractional concentration of mobile charge carriers, assumed to be fully dissociated.

Now with the choice of $\varepsilon_{1}=\varepsilon_{\mathrm{PC}}$ and with $\eta \neq 1$, it follows from Eq. (2) that

$$
\varepsilon_{\mathrm{EM}}^{\prime} / \varepsilon_{0} \rightarrow(1+2 \eta) /(1-\eta) \quad \text { as } \omega \rightarrow 0,
$$

and

$$
\varepsilon_{\mathrm{EM}}^{\prime} / \varepsilon_{0} \rightarrow 2(1-\eta) /(2+\eta) \text { as } \omega \rightarrow \infty .
$$

These expressions lead to the plausible results $\varepsilon_{\mathrm{EM}}^{\prime}>\varepsilon_{0}$ for $\omega=0$ and $\varepsilon_{\mathrm{EM}}^{\prime}<\varepsilon_{0}$ for $\omega=\infty$.

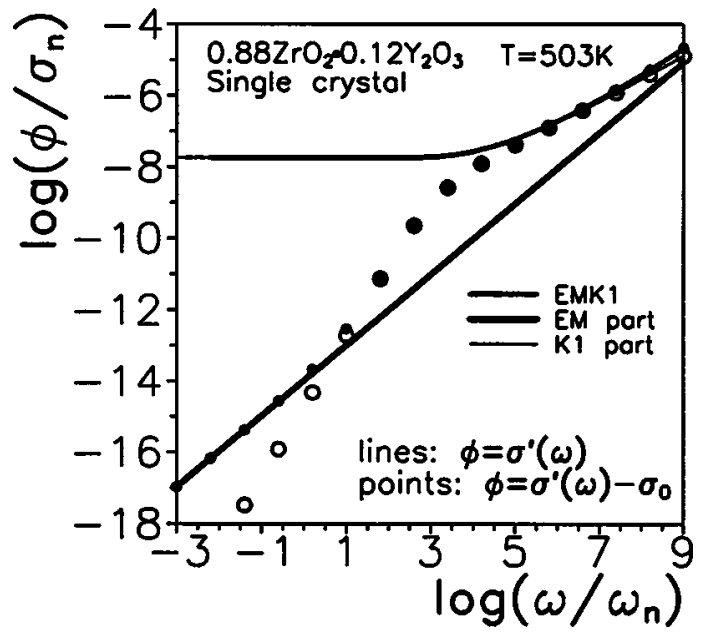

FIG. 1. Dissection of synthetic data derived from fitting experimental $\sigma(\omega)$ data with the EMK1 composite model for the choice $\eta=0.12$. Real part conductivity results are compared for the full EMK1 model, the conductivesystem K1 part, and the dielectric-system effective-medium part. Open circles are for the K1 part and closed circles are for the EMK1. Here and elsewhere $\omega_{n}=1 \mathrm{r} / \mathrm{s}$ and $\sigma_{n}=1 \mathrm{~S} / \mathrm{cm}$.

\section{DATA FITTING AND EFFECTIVE-MEDIUM BEHAVIOR}

\section{A. Conductive-system response dominant}

We begin with an analysis of single-crystal $0.88 \mathrm{ZrO}_{2} \cdot 0.12 \mathrm{Y}_{2} \mathrm{O}_{3}$ data at $T=503 \mathrm{~K}$. Frequency-response data for this material at various temperatures have already been analyzed with the CK1S, PK1, PK1S, and CPK1 models. ${ }^{7,12,26}$ The SCPE response element, represented here by $\mathrm{S}$, may be expressed by $\sigma_{\mathrm{SC}} \equiv \varepsilon_{V} A_{\mathrm{SC}}(i \omega)^{\gamma_{\mathrm{SC}}}$. It has frequently been used to represent blocking or partially blocking electrode effects with $\gamma_{\mathrm{SC}}$ equal to or slightly smaller than unity, respectively. ${ }^{5,7,12,23,31}$ For the present $T=503 \mathrm{~K}$ data set, no series element was needed, and using an EMK1 model leads to the excellent fit value of $\mathrm{S}_{F}$, the relative standard deviation of the fit, of 0.00525 , slightly smaller than that obtained earlier using the PK1 model. ${ }^{7}$ The CNLS fit was carried out using LEVM with proportional weighting at the complex conductivity level.

Although the fit was very good, the EM parameter $\eta$ could not be accurately estimated from the available data. Simulation results showed that appreciably more accurate data would be needed to estimate it adequately. Therefore, it was fixed at the value of $x_{c}=0.12$ of the present material. In order to generate data that allow accurate subtraction and extrapolation, the well-determined EMK1-fit parameter estimates were rounded off to the following values: K1: $\rho_{0}$ $=1 / \sigma_{0}=5.421 \times 10^{7} \Omega \mathrm{cm}, \tau_{0}=4 \times 10^{-6} \mathrm{~s}$, and $\beta_{1}=1 / 3$; and EM: $\varepsilon_{0}=\varepsilon_{D \infty}=23, A_{\mathrm{PC}}=32.3$, and $\gamma_{\mathrm{PC}}=0.024$. These values may then be used with LEVM to generate widefrequency-range data consistent with the measured results in their common range.

Figure 1 shows wide-range results, using the above parameters, for $\sigma^{\prime}(\omega), \sigma_{S}^{\prime}(\omega)$, and the EM part of $\sigma^{\prime}(\omega)$. The slopes of these curves are shown explicitly in Fig. 2. We see that the $\mathrm{K} 1 \sigma_{S}^{\prime}(\omega)$ slope reaches its proper low-frequencylimiting value of 2 and the EM slope is very close to unity 


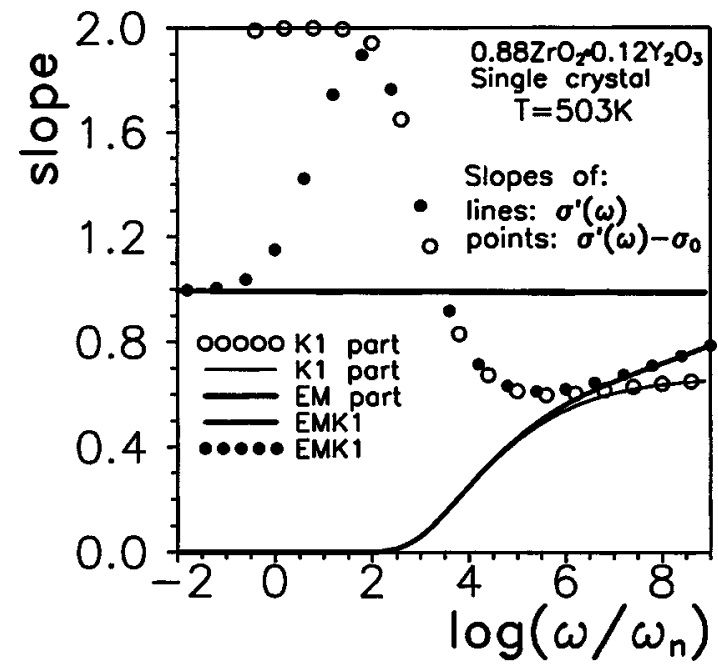

FIG. 2. $\log -\log$ slopes of the real part conductivity responses presented in Fig. 1.

(constant loss) over the entire range shown. It is evident from the results shown in both Figs. 1 and 2 that above $10^{8} \mathrm{r} / \mathrm{s}$ the total response line lies above that arising from the K1 response and its slope approaches that of the EM response. Here, the high-frequency-limiting slope of the $\mathrm{K} 1$ response is $1-\beta_{1}=2 / 3$. Further, we see that the solid $\sigma_{S}^{\prime}(\omega)$ points also closely approach the EM response at sufficiently low frequencies. Thus, the present results indicate that NCL behavior for a given temperature may become dominant in $\sigma_{S}^{\prime}(\omega)$ at both low and high frequencies but not necessarily between these regions where hopping may be dominant.

Although NCL dominance is often identified only for low-temperature data (Refs. 32 and 33, and Refs. 1-6 therein), many low-temperature ${ }^{8,11}$ or even aboveroom-temperature ${ }^{15}$ data sets involve a crossover transition from K1-type conductive-system response to higherfrequency response with a larger, nearly constant slope of unity or slightly less. Note that the CK1S model can fit lowtemperature experimental data well and exhibits a highfrequency slope appreciably greater than unity, and such fitting leads to a slope estimate of about 1.7 for the same material cited in Fig. 1 at $302 \mathrm{~K}^{12}$ When the observed highfrequency slope is just below unity, however, it has been shown that either the CK1S or the PK1 model can often fit such data equally well, and they can only be differentiated by their different responses at sufficiently low frequencies. ${ }^{12}$ It is therefore important to distinguish between the nearly full blocking electrode behavior described by the CK1S with 1 $-\gamma_{\mathrm{SC}} \ll 1$ and PK1 or EMK1 NCL response with $\gamma_{\mathrm{PC}} \ll 1$, a task best carried out using CNLS fitting. Unfortunately, no such tests were applied for the data sets in Refs. 8, 11, and 15 that showed crossover.

Let us now consider EM behavior at the epsilon level calculated directly from the EM parameters and thus not involving such a conductive-system dielectric parameter as $\varepsilon_{C 1 \infty}$. Since we have proposed that for the EM $\varepsilon_{1}$ $=\varepsilon_{\mathrm{PC}}(\omega)$, a quantity arising from interactions between bulk matrix quantities and vibrating ions, or even from forwardbackward ionic hopping at lower frequencies, the present

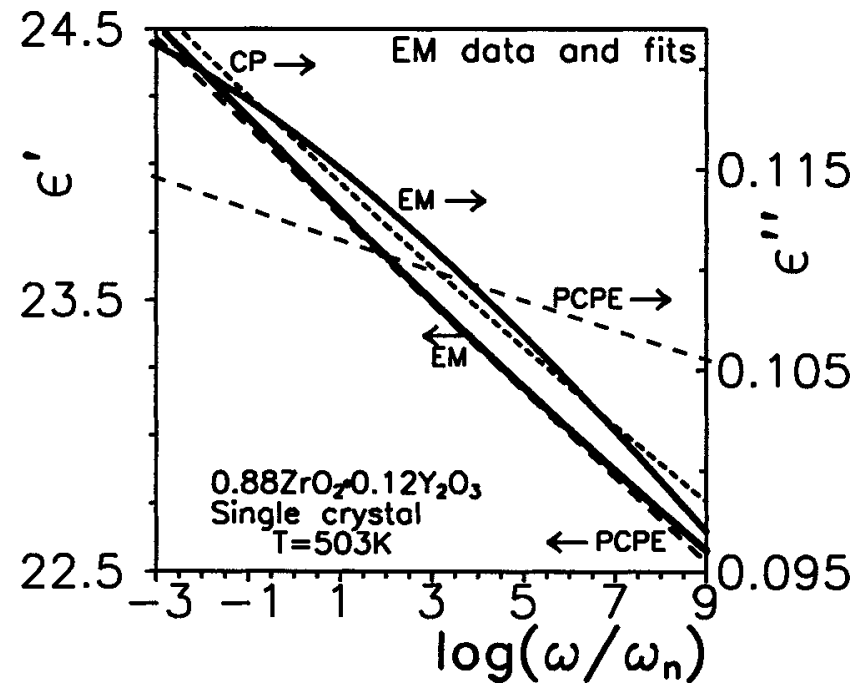

FIG. 3. Dielectric-level $\varepsilon^{\prime}(\omega)$ and $\varepsilon^{\prime \prime}(\omega)$ EM parts of the synthetic data. The lines marked CP and PCPE are fits of the EM responses using the composite CP model and the PCPE model.

EM bulk dielectric response is driven in part by ion motion and only becomes independent of conductive-system response when $\eta=0$ and, as shown by Eqs. (3) and (4), then $\varepsilon_{\mathrm{EM}}^{\prime}=\varepsilon_{0}=\varepsilon_{D_{\infty}}$.

Figure 3 shows both $\varepsilon^{\prime}(\omega)$ and $\varepsilon^{\prime \prime}(\omega)$ results, not only for the EM but also for those obtained from $\mathrm{CP}$ and $\mathrm{P}$ (PCPE) fits of the EM data. Clearly, the three-parameter CP fit yields results much closer to the EM lines than does the two-parameter PCPE, and the real part of the CP fit (not shown) is appreciably closer to the $\varepsilon^{\prime}(\omega)$ EM line than is its imaginary part. Some numerical results for the EM data and fits are shown in Table II. It is clear that the EM parameter values are not entirely comparable to those obtained from the two fits. The EM $\varepsilon_{D \infty}$ value in the absence of ionic effects, 23 , may be compared to the $\mathrm{CP}$ approximate estimate of $(14.83+9.16)=23.99$ and the PCPE estimate of $A_{\mathrm{PC}}$ $=23.97$. The Fig. 3 results show that $\varepsilon^{\prime}$ varies with frequency sufficiently over the 12 frequency decades in Fig. 3 so that none of these constant values is entirely representative. Further, the other fit values of $\gamma_{\mathrm{PC}}$ are much smaller than the $\mathrm{EM}$ one even though the $\varepsilon^{\prime}$ frequency dependencies are somewhat comparable. Because of the small size of the EM $\gamma_{\mathrm{PC}}$ parameter, very much lower frequencies would be required to show the final approach of $\sigma_{S}^{\prime}(\omega)$ to the limiting response following from Eq. (3).

TABLE II. Dielectric constant results from fitting the complex EM data derived from the full EMK1 fit of Fig. 1. Here $\eta=0.12$. The $S^{\prime}$ and $S^{\prime \prime}$ quantities are the log-log slopes of the real and imaginary parts of the $\varepsilon(\omega)$ response at the low $(L)$ and high $(H)$ frequency ends.

\begin{tabular}{lcccccc}
\hline \hline Model & $100 \mathrm{~S}_{F}$ & $\varepsilon_{x}$ & $A_{\mathrm{PC}}$ & $\gamma_{\mathrm{PC}}$ & $-100 \mathrm{~S}_{H}^{\prime}$ & $\begin{array}{r}-100 \mathrm{~S}_{L}^{\prime \prime} \\
-100 \mathrm{~S}_{H}^{\prime \prime}\end{array}$ \\
\hline $\mathrm{EM}$ & $\ldots$ & 23 & 32.3 & 0.024 & 0.3148 & 0.4436 \\
& & & & & 0.2735 & 0.1144 \\
$\mathrm{CP}$ & 0.54 & \multirow{2}{*}{14.83} & 9.16 & 0.0081 & 0.3198 & 0.8092 \\
& & & & & 0.2777 & 0.8093 \\
$\mathrm{PCPE}$ & \multirow{2}{*}{3.04} & $\ldots$ & 23.97 & 0.002981 & 0.2981 & 0.2981 \\
\hline \hline
\end{tabular}




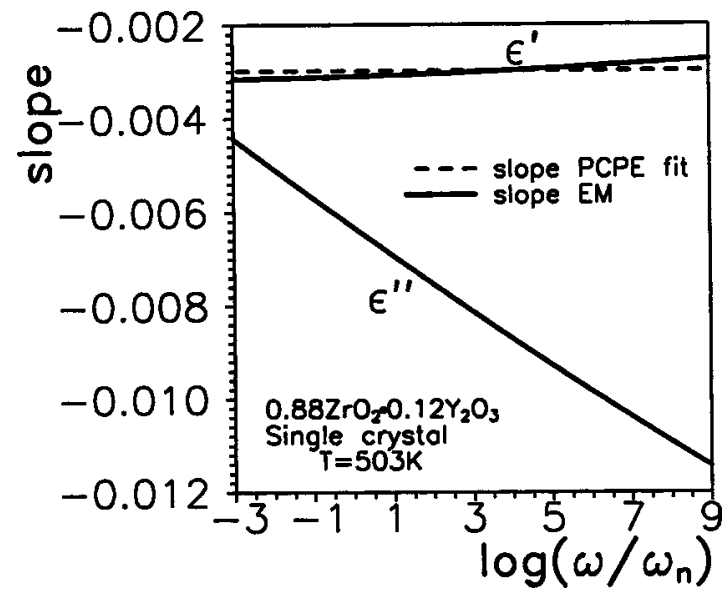

FIG. 4. Log-log slopes of the EM lines and the PCPE fits presented in Fig. 3 . The real and imaginary slopes of the PCPE responses are identical and equal to -0.0031 .

Figure 4 compares the slopes of the EM and PCPE results in Fig. 3. The slopes of the real and imaginary PCPE parts are the same, and are quite different from those of the EM. Clearly, the parameters of the PCPE that is a part of the EM model are appreciably different from those obtained by fitting the EM data with a single PCPE. Finally, it is evident from Figs. 3 and 4 that the EM $\varepsilon^{\prime \prime}(\omega)$ response is of variable NCL character but not of exact constant-loss character.

\section{B. Nearly constant-loss response dominant}

In 1998, Nowick et al. presented frequency response data for a $\mathrm{Na}_{2} \mathrm{O} \cdot 3 \mathrm{SiO}_{2}$ glass over the range from 2 to 435 $\mathrm{K} .{ }^{2}$ Unfortunately, the original data are unavailable, but those at $122 \mathrm{~K}$, presented in their Fig. 8, show $\varepsilon^{\prime}(\omega)$ power-law response that can be accurately fitted by a PCPE (or SCPE) response function. ${ }^{5}$ The $\varepsilon^{\prime \prime}(\omega)$ power-law response following from this fit disagreed with the Ref. 2 experimental results for this quantity, ones that were very close to being independent of frequency over more than two decades: essentially constant loss! An explanation for this discrepancy was proposed in Ref. 5 by considering the response of a pure capacitance or dielectric constant in series with a PCPE or SCPE model. Such a composite model is the PC, a series combination of the PCPE and a capacitance that might possibly model complete blocking behavior at the electrodes of the material. As shown in Ref. 5, the PC model can indeed lead to a very close approximation of constant loss over an appreciable range of frequency.

Nevertheless, the case against the presence of actual constant loss over a finite frequency region is made in Refs. 5 and 34-36. These results thus raise the question of how well the EM response model might be able to reproduce the effects found in Ref. 2, either with or without additional series capacitance present. To obtain synthetic data that duplicate as closely as possible the Ref. $2 T=122 \mathrm{~K}$ results, we started with the PCPE power-law fit of the exact $\varepsilon^{\prime}(\omega)$ data of Refs. 2 and 5 and generated a full complex PCPE response from it. Then PC fits of this data set were carried out with different values of series capacitance. The value of the specific capacitance that led to the closest approximation to
TABLE III. Synthetic $\varepsilon(\omega)$ data and fit parameters for $\mathrm{Na}_{2} \mathrm{O} \cdot 3 \mathrm{SiO}_{2}$ glass at $122 \mathrm{~K}$ (after Ref. 2) using the PCPE, PC, and EM composite response models. Row 2 shows an EM fit of the exact data of row 1, while rows 4-7 are fits of the row 3 exact data. For the EM fits, $\eta$ was held fixed at 0.25 . Quantities such as 1.54 designate the values of series specific capacitance in $\mathrm{pF} / \mathrm{cm}$.

\begin{tabular}{llcccc}
\hline \hline Row & \multicolumn{1}{c}{ Model } & \multicolumn{1}{c}{$\mathrm{S}_{F}$} & $A_{\mathrm{PC}}$ & $\gamma_{\mathrm{PC}}$ & $\varepsilon_{D \infty}$ \\
\hline 1 & PCPE & $\ldots$ & 8.885 & 0.00310 & $\ldots$ \\
2 & EM & $4.86 \times 10^{-5}$ & 14.31 & 0.01157 & 7.497 \\
3 & PC-1.54 & $\cdots$ & 18.15 & 0.00617 & $\ldots$ \\
4 & PC-1.00 & $7.91 \times 10^{-3}$ & 41.32 & 0.01325 & $\ldots$ \\
5 & EMC-1.00 & $9.90 \times 10^{-4}$ & 68.08 & 0.05066 & 34.80 \\
6 & EMC- 1.54 & $1.93 \times 10^{-4}$ & 31.37 & 0.02307 & 14.93 \\
7 & EM & $4.51 \times 10^{-5}$ & 21.54 & 0.01239 & 6.500 \\
\hline \hline
\end{tabular}

frequency-independent $\varepsilon^{\prime \prime}(\omega)$ response was found to be 1.54 $\mathrm{pF} / \mathrm{cm}$. The resulting PC-1.54 fit parameters were then used to generate exact PC-1.54 data. These PC results satisfy the Kronig-Kramers relations, which is not true for real-part power-law response with its imaginary part completely frequency independent.

Table III shows in rows 1 and 3 the parameter values used for generating the exact PCPE and PC-1.54 data sets. In rows 4-7 PC and EM fitting results of the PC-1.54 data are presented for various values of series capacitance. It is evident from the results of rows 4 and 5 that, for a value of 1.00 $\mathrm{pF} / \mathrm{cm}$, the EMC model yields a much better fit than does the PC model. Also, although the EMC-1.54 model leads to an even better fit, the fit in row 7 using the EM model alone is appreciably better. Its $\varepsilon_{D \infty}$ estimate of 6.50 is smaller than the $\varepsilon_{\infty}$ value of 9.45 listed in Ref. 2 for higher temperatures, but since the EM parameters in row 7 lead to exceptionally close fits of both the real and imaginary parts of the data, one might well conclude that the 6.50 value is the more appropriate. Incidentally, this value and $\eta=0.25$ lead, using Eqs. (3) and (4), to the estimates $\varepsilon_{\mathrm{EM}}^{\prime}(0)=13$ and $\varepsilon_{\mathrm{EM}}^{\prime}(\infty)$ $=13 / 3$. Thus, even if an estimate of the latter quantity could be obtained, it should not be identified as $\varepsilon_{\infty}=\varepsilon_{D \infty}$ if the EM model best represents the data.

Comparison of the EM row 2 and row 7 corresponding values demonstrates the important result that the EM model can equally well fit data for which the $\varepsilon^{\prime \prime}(\omega)$ response either is of power-law form or is very nearly independent of the frequency. The former situation was investigated in Sec. III A, while we are primarily interested here in response of the latter form. ${ }^{2}$

Figure 5 shows $\varepsilon^{\prime}(\omega)$ PCPE and PC data and fits, and Fig. 6 presents the corresponding $\varepsilon^{\prime \prime}(\omega)$ results. Although not shown in Fig. 5, the original PCPE $\varepsilon^{\prime}(\omega)$ data cannot be distinguished there from that of the PC-1.54 response although they are not exactly the same. Further, Fig. 5 shows that the real part of the complex $\varepsilon(\omega)$ EM fit of the PC-1.54 data, as well as that of an EMC-1.00 fit, also cannot be distinguished in the graph from the original data. This is clearly not the case for the PC-1.00 fit results.

Figure 6 compares the PCPE data and EM fit with those for the PC-1.54 data set and various fits of it. The PC-1.54 $\varepsilon^{\prime \prime}(\omega)$ nearly horizontal data line varies continuously over the range shown but is nevertheless constant to four signifi- 


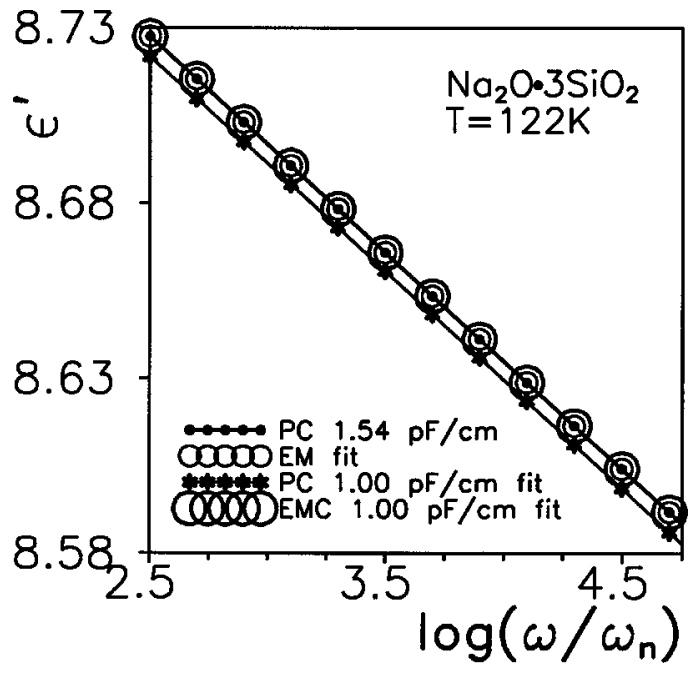

FIG. 5. $\varepsilon^{\prime}(\omega)$ results for the exact PC-1.54 data set and various fits to it. The EM fit is that in row 7 of Table III.

cant figures. We see, in agreement with the Table III results that, although the EMS-1.54 fit is excellent, the EM one is appreciably superior. Again, the PC-1.00 fit is particularly poor.

The EM fit of the PC-1.54 complex $\varepsilon(\omega)$ data is superior to the EMC-1.54 fit and leads to a more plausible estimate of $\varepsilon_{D \infty}$ than does the latter. Further, there is difficulty in justifying the values of series capacitance used here in the PC and EMC models. They cannot be identified with the conductive system $\varepsilon_{C 1 \infty}$ quantity because $\varepsilon_{C 1}(\omega)$ is in parallel, not in series, with the PCPE or EM dielectric response models. Although electrode effects are indeed in series, the usual double-layer capacitance associated with the blocking of mobile charge at the electrodes is generally three or more orders of magnitude larger than $1.54 \mathrm{pF} / \mathrm{cm}$. With a series specific capacitance for the EMC even two orders of magnitude larger, negligible difference is found here between the EM model (with series capacitance of infinite size yielding no effect) and such an EMC model. Thus, even if electrodeblocking capacitances are significant for the present material

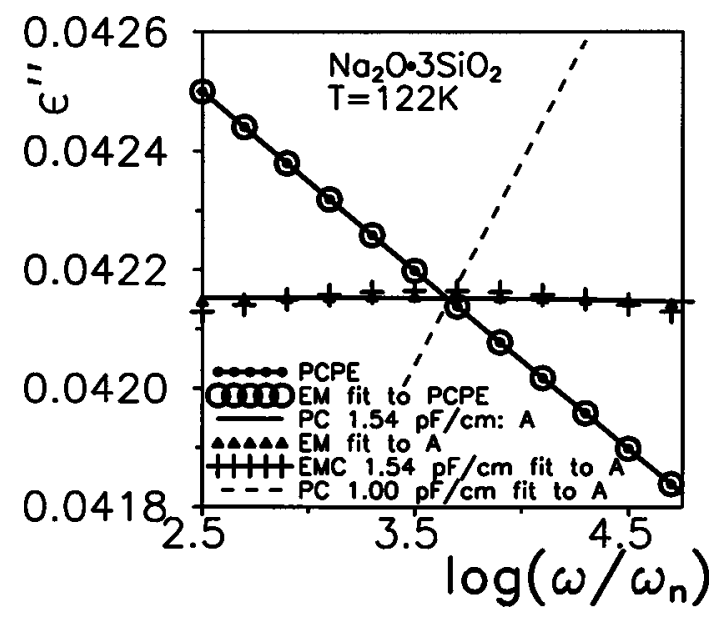

FIG. 6. Comparison of $\varepsilon^{\prime \prime}(\omega)$ data and fits for the PCPE and PC-1.54 models.

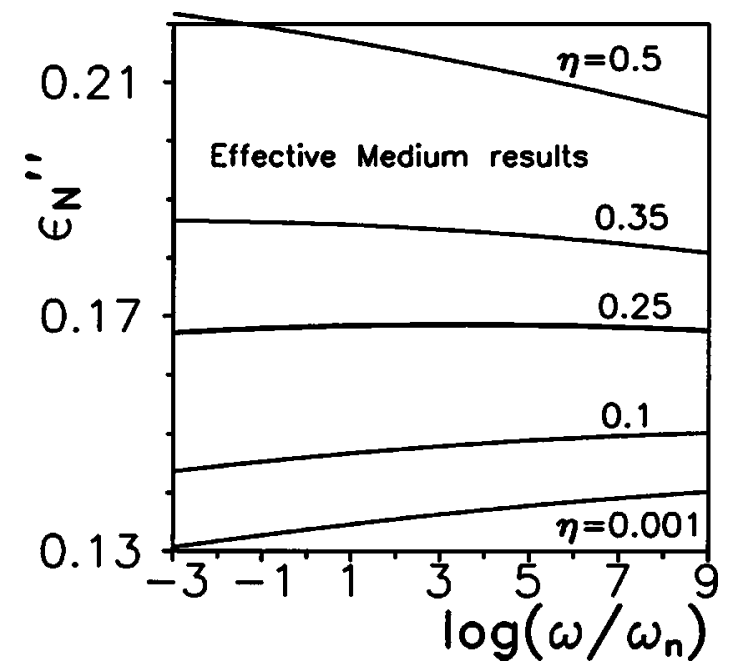

FIG. 7. Dependence of $\varepsilon_{N}^{\prime \prime}(\omega) \equiv \varepsilon^{\prime \prime}(\omega) / \eta$ on $\eta$ for a wide frequency range. All the other parameters of the EM fit in row 7, Table III, are held constant for these calculations.

at $122 \mathrm{~K}$, they seem unlikely to explain the small value of $1.54 \mathrm{pF} / \mathrm{cm}$. Therefore, an appreciable advantage of the EM model over the EMC or PC ones is that it can lead very closely to constant-loss behavior without the need for the presence of any small series capacitance.

Figure 7 demonstrates how the EM $\varepsilon^{\prime \prime}(\omega)$ response varies over a very wide frequency range for different $\eta$ values when the parameters of Table III, row 7 remain constant. Evidently, the slopes of the $\varepsilon^{\prime \prime}(\omega)$ curves can be either positive or negative, although they are not completely constant over the present frequency range. For example, for the $\eta$ $=0.001$ curve, the $\varepsilon^{\prime \prime}(\omega)$ slope varies from about 3.5 $\times 10^{-3}$ at the low-frequency end to about $1.5 \times 10^{-3}$ at the high-frequency end of the range. The corresponding $\varepsilon^{\prime}(\omega)$ slope is of the order of $10^{-5}$ because for such a small value of $\eta$ the $\varepsilon^{\prime}(\omega)$ response is very close to that of the constant $\varepsilon_{D \infty}$ parameter. For $\eta=0.5$, on the other hand, the $\varepsilon^{\prime \prime}(\omega)$ slope is about $-4 \times 10^{-3}$ at the high-frequency end. Although these results are interesting, one expects that most, if not all, of the EM parameters may depend on $\eta,{ }^{7}$ so in practice the EM may not actually lead to positive $\varepsilon^{\prime \prime}(\omega)$ slopes and thus to ones for the real part of the conductivity that slightly exceed unity.

\section{DISCUSSION OF VARIOUS RESPONSE MODELS}

Recently Ngai and León stated, "One of the frontiers of research in the field of dynamics of ions in ionic conductors is the origin of the ubiquitous near constant loss (NCL) ...," ${ }^{37}$ thus underscoring the need to develop a realistic model for this phenomenon. They also mentioned that the presence of NCL in crystalline ionic conductors “. . . rules out the possibility that the NCL of present interest originates from the glassy matrix in which the ions are embedded." This conclusion is, however, inapplicable to the dielectric NCL model discussed earlier and its present EM development, both of which quantitatively model NCL in both glassy or crystalline materials very well. ${ }^{7}$ These approaches identify NCL behavior as being induced in the bulk 
dielectric matrix by the presence and motion of ions in the material, not as originating as an independent bulk effect.

Noteworthy, however, is the "jellyfish" model of Lu and Jain, 1,13,38 one partly based on an asymmetric double-well potential that involves transitions of ions from one well to the other. Most comparisons of this approach with experiment have, however, involved the empirical equation,

$$
\sigma^{\prime}(\omega, T)=A \omega^{\beta} \exp \left(\alpha^{\prime} T\right)
$$

where $1.00 \leqslant \beta \leqslant 1.13$ and $\alpha^{\prime}$ is taken temperature independent and very small. This is not a full response model, however, both because no corresponding expression for $\sigma^{\prime \prime}(\omega, T)$ is provided and because it does not include any separate diffusive ion-hopping response and a transition from that to power-law-frequency response. It thus applies only at sufficiently low temperatures that hopping effects are negligible. ${ }^{13,38}$ It is important to mention that in this temperature region the asymmetric-double-well-potential model, unlike that of Eq. (5), can explain the appearance of an interesting peak in $T \sigma_{0}(T)$ vs $\mathrm{T}$ observed for some glasses at low ionic concentrations. ${ }^{38}$

In Refs. 13 and 38 an interesting physical picture of the origin of such response is proposed, one that involves the collective motion of a jellyfish group of glass-network atoms and ions (called ions in the Ref. 14 mention of this approach). Although this model is not strictly of usual NCL character since $\beta$ is not expected to be less than unity, it could be readily identified as such if experimental analysis yielded a somewhat uncertain estimate of $\beta$.

It is worth mentioning that exponential temperature response like that in Eq. (5) was later employed for NCL temperature dependence by others ${ }^{11}$ and found to be superior to an Arrhenius expression. Later work of the present author suggested, however, that the reverse conclusion was preferable, at least for the data considered there. ${ }^{16}$ The principal temperature dependence of the EM model is associated with that of the $A_{\mathrm{PC}}$ parameter of Eq. (1), dependence that appears to be very small ${ }^{7}$ and may possibly be best represented by the exponential form above or by an Arrhenius response with very small activation energy. Once a plausible $A_{\mathrm{PC}}(T)$ expression has been established by data fitting over a range of temperatures, it may be used to both fit experimental data or predict NCL temperature dependence provided $\gamma_{\mathrm{PC}}(T)$ is temperature independent, as is the $\beta$ of Eq. (5).

There is general agreement that NCL response is not a significant part of low-frequency hopping behavior, such as that often represented by "universal dynamic response", 2,25 or by the present $\mathrm{K} 1$ model, and Rivera and co-authors ${ }^{14}$ have recently stated, "At this time it is too early to identify the true source of NCL." Nevertheless, a proposal for the origin of NCL behavior has been described, a qualitative approach that assumes that NCL is associated with ions vibrating in potential-well cages that very slowly decay over

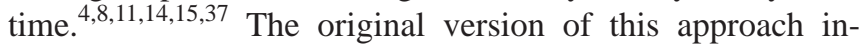
volved the assumption that there is a thermally activated crossover transition from NCL to diffusive response, with NCL ceasing when conductive-system hopping behavior begins. ${ }^{4,8,11,14}$ In later work on other materials, ${ }^{15,37}$ the authors defined a finite-length crossover region from hopping to NCL response with somewhat qualitatively determined boundaries, and no discussion was provided concerning possible types of charge motion present in this intermediate frequency region, one of a decade or more in extent. Further, their approach involved fitting the hopping part of the response by means of the OMF K1 model, one identified in Sec. II A as inappropriate.

The above qualitative model is characterized as serial and thus involves neither parallel nor series response, a necessary justification for its proposed physical explanation of crossover. In fact, the authors claimed in Refs. 8 and 11 that a composite response model such as the "so-called augmented Jonscher form,", 25

$$
\sigma^{\prime}(\omega)=\sigma_{0}\left[1+\left(\omega / \omega_{0}\right)^{n}\right]+A \omega,
$$

fails to describe ac conductivity in the whole range of frequency and temperature. This real-part model is of parallel form and involves an $A \omega$ constant-loss term.

Detailed quantitative analysis of synthetic data shows, however, that a parallel model can indeed describe well both the real and imaginary parts of much experimental ac conductivity data, including the details of crossover. ${ }^{5,12,16}$ In a parallel model, it is unnecessary to make the questionable serial-response assumption that NCL and ionic-hopping effects cannot exist simultaneously. Instead, the quantitative parallel approach, as in the PK1 or EMK1 models, indicates that NCL behavior rapidly becomes less and less significant (compared to hopping effects described by the K1 model) at frequencies appreciably below crossover, and K1 effects are dominated by the NCL response at frequencies appreciably above crossover. Finally, the cage-decay, serial NCL model is incomplete since no analytical expression is available for its complex conductivity that encompasses the transition from hopping to serial behavior. In contrast, the EMK1 model appears to be the only one so far that provides such a fully complex physically realizable expression that can be used for CNLS fitting of data that exhibit both hopping and NCL or either one separately.

\section{SUMMARY}

A physically realizable NCL response model, based on effective-medium physics, was described and its response possibilities investigated. This model, designated the EM, is able to fit a wide variety of NCL-type frequency response, ranging from that of a complex power law, the PCPE model, to that where the real part of the NCL dielectric constant is experimentally indistinguishable from power-law behavior but its imaginary part is very nearly frequency independent: constant loss behavior. It is suggested that EM response involves electrical coupling between vibrating and even hopping ions and dipoles of the bulk material. Other NCL response models considered herein are characterized as inappropriate or incomplete and do not allow CNLS fitting of complex frequency-response data that encompass the transition from diffusive hopping behavior to NCL response, as does the composite EMK1 model proposed and investigated here. 


\section{ACKNOWLEDGMENTS}

It is a pleasure to thank Dr. A. S. Nowick and Dr. H. Jain for insightful and helpful discussions. Dr. Carlos León kindly provided the single-crystal data mentioned in Sec. III A.

${ }^{1}$ X. Lu and H. Jain, J. Phys. Chem. Solids 55, 1433 (1994).

${ }^{2}$ A. S. Nowick, A. V. Vaysleyb, and W. Liu, Solid State Ionics 105, 121 (1998).

${ }^{3}$ K. L. Ngai, J. Chem. Phys. 110, 10576 (1999).

${ }^{4}$ C. León, A. Rivera, A. Varez, J. Sanz, J. Santamaria, and K. L. Ngai, Phys. Rev. Lett. 86, 1279 (2001).

${ }^{5}$ J. R. Macdonald, J. Chem. Phys. 115, 6192 (2001).

${ }^{6}$ B. Roling, C. Martiny, and S. Murugavel, Phys. Rev. Lett. 87, 085901 (2001).

${ }^{7}$ J. R. Macdonald, J. Chem. Phys. 116, 3401 (2002).

${ }^{8}$ C. León, A. Rivera, A. Varez, J. Sanz, J. Santamaria, C. T. Moynihan, and K. L. Ngai, J. Non-Cryst. Solids 305, 88 (2002).

${ }^{9}$ C. León, A. Rivera, J. Santamaria, C. T. Moynihan, and K. L. Ngai, Phys. Rev. Lett. 89, 079601 (2002).

${ }^{10}$ B. Roling, C. Martiny, and S. Murugavel, Phys. Rev. Lett. 89, 079602 (2002).

${ }^{11}$ A. Rivera, C. León, J. Sanz, J. Santamaria, C. T. Moynihan, and K. L. Ngai, Phys. Rev. B 65, 224302 (2002).

${ }^{12}$ J. R. Macdonald, J. Non-Cryst. Solids 307-310, 913 (2002).

${ }^{13}$ H. Jain, S. Krishnaswami, and O. Kanert, J. Non-Cryst. Solids 307-310, 1017 (2002).

${ }^{14}$ A. Rivera, J. Santamaria, C. León, J. Sanz, C. P. E. Varsamis, G. D. Chryssikos, and K. L. Ngai, J. Non-Cryst. Solids 307-310, 1024 (2002).

${ }^{15}$ K. L. Ngai, R. W. Rendell, and C. León, J. Non-Cryst. Solids 307-310, 1039 (2002).

${ }^{16}$ J. R. Macdonald, Phys. Rev. B 66, 064305 (2002).
${ }^{17}$ J. R. Macdonald and L. D. Potter, Jr., Solid State Ionics 23, 61 (1987); J. R. Macdonald, J. Comput. Phys. 157, 280 (2000). The newest Windows version of the comprehensive LEVM CNLS fitting program may be downloaded at no cost from http://www.physics.unc.edu/ macd/. It includes an extensive manual, executable programs, and full source code. More information is provided about LEVM at this www address.

${ }^{18}$ J. C. Dyre and T. B. Schrøder, Rev. Mod. Phys. 72, 873 (2000).

${ }^{19}$ C. T. Moynihan, L. P. Boesch, and N. L. Laberge, Phys. Chem. Glasses 14, 122 (1973).

${ }^{20}$ J. R. Macdonald, J. Non-Cryst. Solids 197, 83 (1996).

${ }^{21}$ J. R. Macdonald, J. Non-Cryst. Solids 212, 95 (1997).

${ }^{22}$ J. R. Macdonald, Phys. Rev. B 63, 052205 (2001).

${ }^{23}$ J. R. Macdonald, J. Appl. Phys. 90, 153 (2001).

${ }^{24}$ J. R. Macdonald, Solid State Ionics 150, 263 (2002).

${ }^{25}$ J. R. Macdonald, Solid State Ionics 133, 79 (2000).

${ }^{26}$ J. R. Macdonald, J. Chem. Phys. 118, 3258 (2003).

${ }^{27}$ Impedance Spectroscopy-Emphasizing Solid Materials and Systems, edited by J. R. Macdonald (Wiley-Interscience, New York, 1987); pp. 3940.

${ }^{28}$ D. R. McKenzie and R. C. McPhedran, Nature (London) 265, 128 (1977).

${ }^{29}$ C. Levy and D. Stoud, Phys. Rev. B 56, 8035 (1997).

${ }^{30}$ R. F. Bianchi, G. F. Leal Ferreira, C. M. Lepienski, and R. M. Faria, J. Chem. Phys. 110, 4602 (1999).

${ }^{31}$ T. Pajkossy, J. Electroanal. Chem. 364, 111 (1994).

${ }^{32}$ J. R. Macdonald, J. Non-Cryst. Solids 210, 70 (1997).

${ }^{33}$ O. Kanert, R. Küchler, P. C. Soars, Jr., and H. Jain, J. Non-Cryst. Solids 307-310, 1031 (2002).

${ }^{34}$ J. R. Macdonald, J. Appl. Phys. 75, 1059 (1994).

${ }^{35}$ J. R. Macdonald, Appl. Phys. A: Solids Surf. A59, 181 (1994).

${ }^{36}$ J. R. Macdonald, J. Non-Cryst. Solids 210, 70 (1997).

${ }^{37}$ K. L. Ngai and C. León, Phys. Rev. B 66, 064308 (2002).

${ }^{38}$ H. Jain, Met., Mater. Processes 11, 317 (1999). 